\title{
Disruption of the association between drug transporter and actin cytoskeleton abolishes drug resistance in hypertrophic scar
}

\author{
Linlin Su ${ }^{1, *}$, Lanqing Fu ${ }^{2,}{ }^{*}$, Yan $\mathrm{Li}^{1, *}$, Fangfang Yang ${ }^{1}$, Min Zhang ${ }^{1}$, Dahai Hu ${ }^{1}$ \\ ${ }^{1}$ Department of Burns and Cutaneous Surgery, Xijing Hospital, The Fourth Military Medical University, Xi'an, Shaanxi 710032, \\ China \\ ${ }^{2}$ Department of Orthopedics, Jingzhou Central Hospital, Tongji Medical College of Huazhong University of Science and \\ Technology, Jingzhou, Hubei 434020, China \\ *These authors contributed equally to this work
}

Correspondence to: Linlin Su, email: linlinsu@fmmu.edu.cn Dahai Hu, email: hudhai@fmmu.edu.cn

Keywords: hypertrophic scar, fibroblasts, P-glycoprotein

Received: September 01, 2016

Accepted: November 22, 2016

Published: December 01, 2016

\section{ABSTRACT}

Hypertrophic scar is characterized by the overgrowth of fibroblasts and often considered as a kind of benign skin tumor, thus chemotherapeutic drugs have been used to treat scars. In view of the similarity, this study aims to investigate whether drug resistance in cancer that contributes to the failure of chemotherapy also exists in hypertrophic scar, and what is the possible mechanism. Fibroblasts derived from hypertrophic scar and normal skin tissues were first compared for their resistance to verapamil and etoposide phosphate. Scar fibroblasts showed stronger resistance to both verapamil and etoposide than normal fibroblasts, also scar fibroblasts expressed more P-glycoprotein and MRP1 than normal fibroblasts. When scar fibroblasts were pre-treated with PSC833 or probenecid, a P-glycoprotein or MRP1 inhibitor respectively, the resistance to verapamil or etoposide was strongly attenuated. Moreover, co-immunoprecipitation revealed more association of P-glycoprotein/MRP1 with actin filaments in scar fibroblasts than normal fibroblasts. The resistance in scar fibroblasts to verapamil and etoposide was almost abolished when pre-treated with latrunculin-A or a specific anti-actin antibody. Taken together, this study suggests that the enhanced expression of drug resistance-related transporters and their increased association with actin cytoskeleton contribute to the resistance to chemotherapeutic drugs in hypertrophic scar. Thus, down-regulating the expession of drug transporters or disrupting drug transporter-actin filament interaction might be novel and effective ways for hypertrophic scar treatment.

\section{INTRODUCTION}

Hypertrophic scar (HS) is often considered as a kind of benign skin tumor because of its excessive fibroblast proliferation. A major challenge in cancer treatment is the resistance of cancer cells to chemotherapeutic agents, which is mediated by several drug resistance-related drug transporters [1-2], particularly P-glycoprotein and multidrug resistance-associated protein 1 (MRP1). P-glycoprotein and MRP1 are overexpressed in various tumors [3-4], they exclude therapeutic drugs out of cells and thus decrease the intracellular drug accumulation.
Given that HS shares certain similarity with tumor, this study aims to determine whether drug resistance also exists in HS and whether this is mediated by P-glycoprotein and/ or MRP1.

Recent studies have revealed that the interaction between drug transporter proteins and cytoskeleton system plays essential roles in various cellular processes, such as cross-membrane substance trafficking, intracellular protein transportation, as well as cell signaling [5-7]. Of interest, emerging evidences have suggested an important role of the actin-based cytoskeleton in P-glycoprotein-mediated drug resistance in cancer cells [8]. In fact, the organization 
of actin filaments was involved in the expression and function of P-glycoprotein in osteosarcoma cells that exhibit drug resistance [9]. Cytoskeleton alteration was also found in a human breast cancer cell line that shows drug resistance [10]. Therefore, it is worthy to elucidate whether the interaction between drug transporters, such as P-glycoprotein or MRP1, and actin cytoskeleton would be one of the mechanisms that confer HS resistance.

In this study we have investigated the association between P-glycoprotein/MRP1 and actin, as well as the role of this interaction in P-glycoprotein/MRP1-mediated drug resistance in HS. Our results revealed HS-derived fibroblasts were more resistant to both verapamil and etoposide phosphate than normal fibroblasts. HS overexpressed both P-glycoprotein and MRP1, and the use of P-glycoprotein or MRP1 inhibitor could abolish the drug resistance in HS. Stronger association between P-glycoprotein/MRP1 and actin was found in HS, when latrunculin-A, an actin depolymerization agent, was used to disrupt this association, drug resistance in HS was greatly attenuated. Thus the enhanced P-glycoprotein/ MRP1 expression and their association with actin cytoskeleton might be a potential mechanism conferring HS resistance to chemotherapy, while disrupting this association by well-designed chemicals would be a novel approach for HS treatment.

\section{RESULTS}

\section{Hypertrophic scar-derived fibroblasts were more resistant to both verapamil and etoposide phosphate}

We first investigated the difference on drug resistance between nomal skin and HS. Fibroblasts from normal skin (NF) and hypertrophic scar (HF) were treated with ethanol (vehicle control, VeCtrl), verapamil (Ver, $200 \mu \mathrm{M}$ ), or etoposide phosphate (Etop, $100 \mu \mathrm{M}$ ) for $12 \mathrm{~h}$, cell survival and death were then analyzed by light microscope (Figure 1A) or flow cytometry (Figure 1C). More living cells were observed in HF than in NF after drug treatment (Figure 1A). Prior to Ver or Etop treatment, there were 400 living cells in each group, after drug treatment $\sim 300-340$ living cells left in HF while only $\sim 100$ in NF (Figure 1B). Flow cytometry analysis showed Ver or Etop caused an $\sim 75 \%$ cell death rate in NF while only $\sim 23 \%$ in HF (Figure 1C), consistent with the observation and counting in Figure $1 \mathrm{~A}-1 \mathrm{~B}$.

\section{The protein levels of P-glycoprotein and MRP1 were up-regulated in hypertrophic scar fibroblasts}

Then we studied the expression and localization of two drug resistance-related transporter proteins, namely P-glycoprotein and MRP1, in normal skin and HS. P-glycoprotein and MRP1 showed very few stainings in
NF, while exhibited strong cytoplasm distribution in HF (Figure 2A). The protein level of P-glycoprotein or MRP1 in HF was $\sim 2.5$ - or $\sim 7$-fold higher than that in NF, respectively (Figure 2B). PepT1, an oligopeptide transporter that does not confer cell drug resistance, showed no difference on the staining pattern or expession level between NF and HF and served as an internal control in this study (Figure 2A-2B).

\section{The protein levels of P-glycoprotein and MRP1 were up-regulated in hypertrophic scar dermis in vivo}

In vivo experiments were performed to validate above in vitro data. Dermis from normal skin (ND) and hypertrophic scar (HD) were sectioned and stained with anti-P-glycoprotein, anti-MRP1 or anti-PepT1 antibody for immunohistochemistry (Figure 3A-3B). Results showed only $\sim 10 \%$ P-glycoprotein or $\sim 35 \%$ MRP1-positively stained cells in ND, while $\sim 60 \%$ P-glycoprotein or $\sim 80 \%$ MRP1-positively stained cells in HD (Figure 3A-3B). Immunoblotting analysis further confirmed above observation, the protein levels of P-glycoprotein and MRP1 were up-regulated by $\sim 3-$ or $\sim 7$-fold respectively in HD compared to ND (Figure 3C). In vivo PepT1 distribution or protein expression showed no difference between the two groups (Figure 3A-3C).

\section{Pre-treating cells with P-glycoprotein or MRP1 inhibitor abolished HS drug resistance in vitro}

Next we tried to elucidate whether drug resistance in HS was related to the overexpression of P-glycoprotein or MRP1. PSC833, an inhibitor for P-glycoprotein, and probenecid (Prob), an inhibitor for MRP1, were selected and used in this study. Cells were pre-treated with PSC833 or DMSO (vehicle control) in Figure 4, or pre-treated with Prob or DMSO (vehicle control) in Figure 5 for $12 \mathrm{~h}$, and then subjected to Ver or Etop treatment for 12 h. Light microscopy and flow cytometry analysis revealed that PSC833 (Figure 4) or Prob (Figure 5) pre-treatment significantly attenuated drug resistance in $\mathrm{HF}$, causing rapid decrease on living cell number (Figure 4A-4B and $5 \mathrm{~A}-5 \mathrm{~B}$ ) and increase on dead cell rate (Figures $4 \mathrm{C}$ and 5C). The cytotoxicity of PSC833 and Prob at different concentrations was measured by MTT assay. PSC833 at $5 \mu \mathrm{M}$ (Figure 4D) and Prob at $1 \mathrm{mM}$ (Figure 5D), the concentrations that did not cause cellular toxicity, were used in this study.

\section{The association between P-glycoprotein/MRP1 and actin was up-regulated in hypertrophic scar fibroblasts}

The ultimate question was to reveal the underlying mechanisms that confer HS drug resistance. Recent studies have indicated the involvement of P-glycoprotein- 
cytoskeletal protein (e.g. actin) association in drug transporting protein functions [11]. Therefore, we moved to examine whether the interaction between P-glycoprotein/MRP1 and actin existed in skin fibroblasts, and whether this interaction was different between NF and HF. Co-IP analysis showed NF exhibited weak interaction beetween P-glycoprotein/MRP1 and actin, which was significantly up-regulated in HF (Figure 6A). Dual-labelled immunofluorescent analysis further showed remarkably more co-localization of P-glycoprotein/MRP1 with actin in HF than that in NF (Figure 6B-6C).

The disruption of P-glycoprotein/MRP1-actin association by latrunculin-A abrogated drug resistance in hypertrophic scar fibroblasts

We explored whether disrupting P-glycoprotein/ MRP1-actin association would affect drug resistance
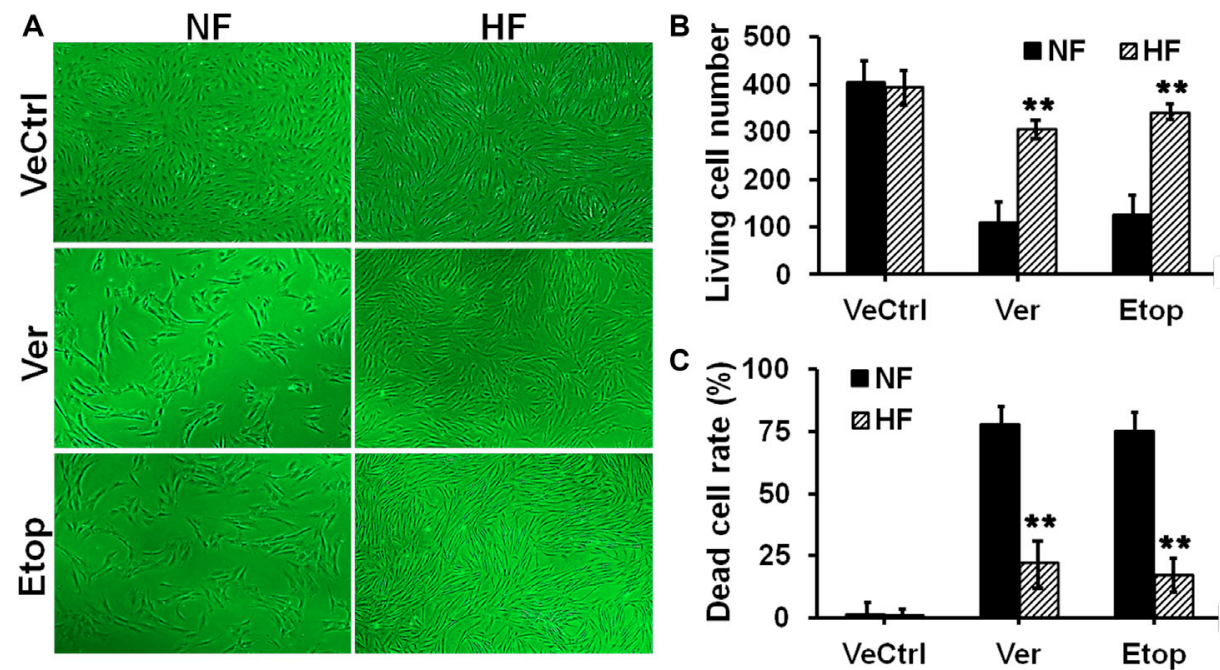

Figure 1: Evaluation of drug resistance in fibroblasts from normal skin and hypertrophic scar. (A) Normal skin fibroblast (NF) and hypertrophic scar fibroblast (HF) were treated with ethanol (vehicle control, VeCtrl), verapamil (Ver, $200 \mu \mathrm{M})$ or etoposide phosphate (Etop, $50 \mu \mathrm{M}$ ) for $12 \mathrm{~h}$, then the cell density in each group was visualized under light microscope. (B) The living cell number under each treatment in (A) was counted and compared between NF and HF. (C) The dead cell rate in each treatment group was analyzed by flow cytometry and compared. Error bars represent means $\pm \operatorname{SD}(n=4),{ }^{*} p<0.01$.
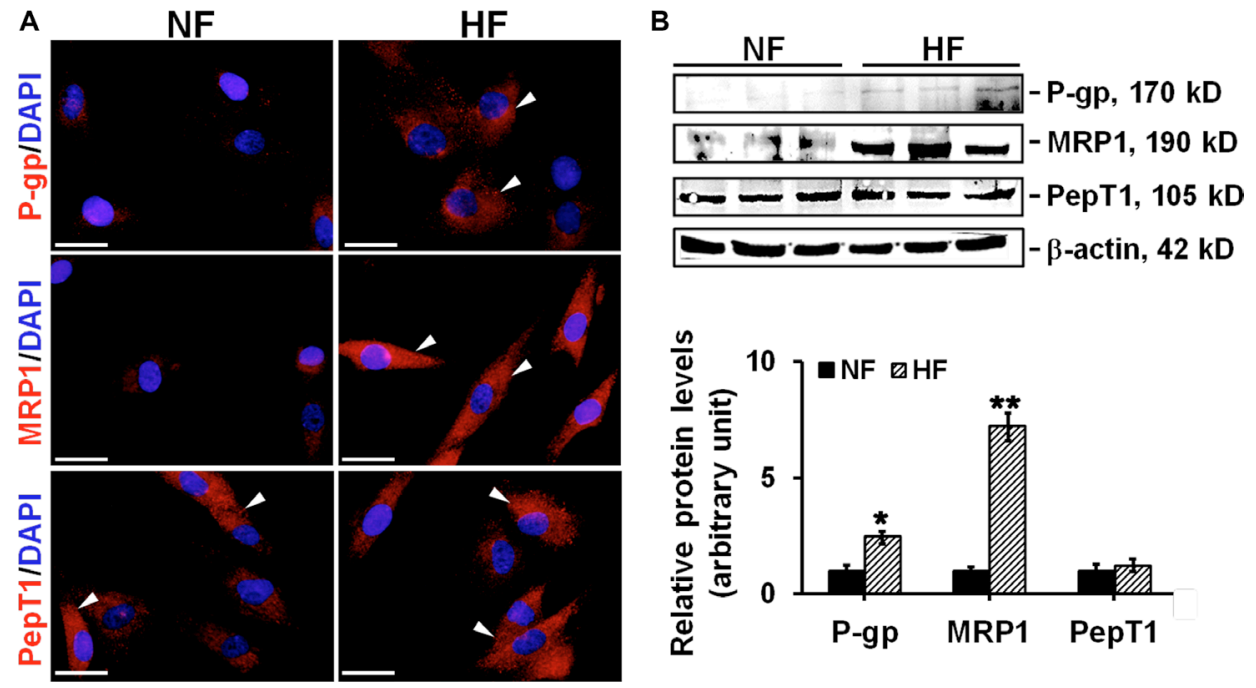

Figure 2: The comparison on the localization and expression of drug transporting proteins in NF and HF. (A) Study by immunocytofluorescence to assess the cellular localization of P-glycoprotein (red), MRP1 (red), and PepT1 (red) in NF and HF. Nuclei were visualized with DAPI (blue). White arrowheads point to the positive immunostainings. Scale bar: $25 \mu \mathrm{m}$. (B) Immunoblotting of P-glycoprotein, MRP1 and PepT1 in the lysates of NF and HF. $\beta$-actin served as an equal protein loading control. Histogram compared the difference on the relative protein expression between NF and HF. Each data point was normalized against corresponding $\beta$-actin with the average value after normalization in NF arbitrarily set as 1 . Error bars represent means \pm SD $(n=6),{ }^{*} p<0.05,{ }^{* *} p<0.01$. 

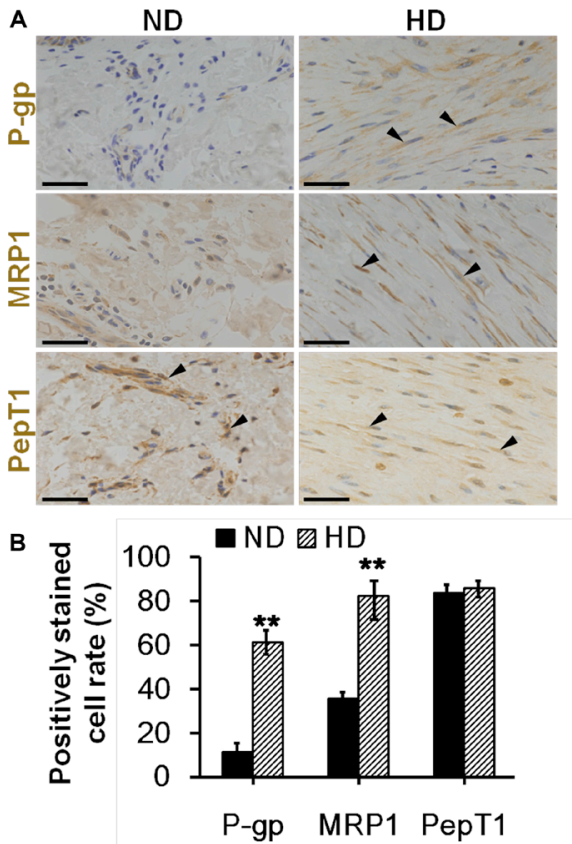

C
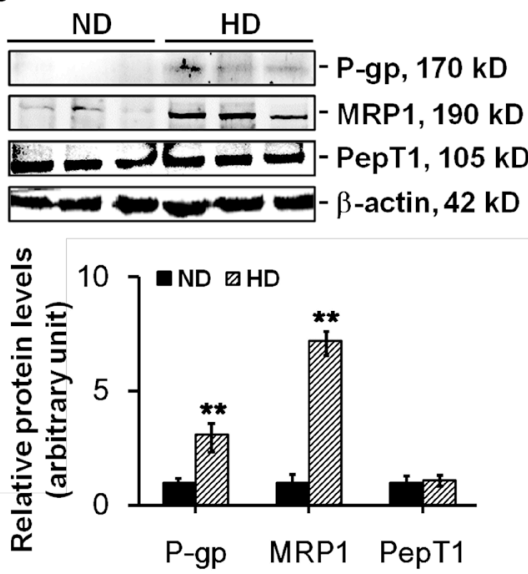

Figure 3: The comparison on the distribution and expression of drug transporting proteins between normal dermis and scar dermis in vivo. (A) Study by immunohistochemistry to assess the distribution of P-glycoprotein (brownish), MRP1 (brownish), and PepT1 (brownish) in normal dermis (ND) and hypertrophic scar dermis (HD). Nuclei were conterstained with hematoxylin (blue). Black arrowheads point to the positive immunostainings. Scale bar: $50 \mu \mathrm{m}$. (B) Histogram comparing the P-glycoprotein-, MRP1-, or PepT1-positively stained cell rate between ND and HD. (C) Immunoblotting of P-glycoprotein, MRP1 and PepT1 in the lysates from ND and HD. $\beta$-actin served as an equal protein loading control. Histogram compared the difference on the relative protein expression between $\mathrm{ND}$ and HD. Each data point was normalized against corresponding $\beta$-actin with the average value after normalization in NF arbitrarily set as 1 . Error bars represent means $\pm \mathrm{SD}(n=6), * * p<0.01$.

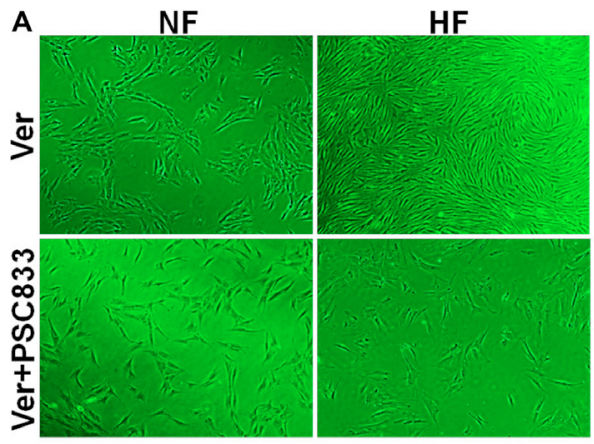

B

C

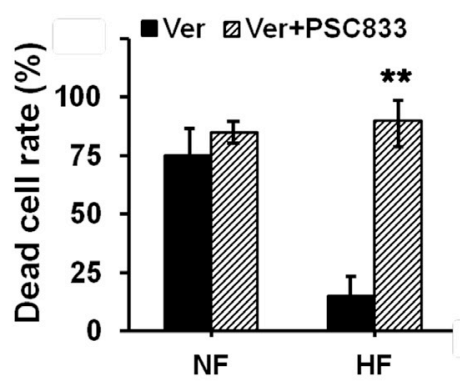

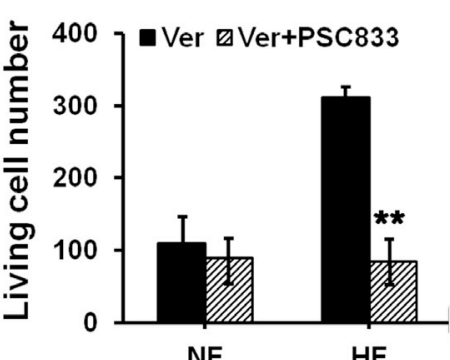

NF HF

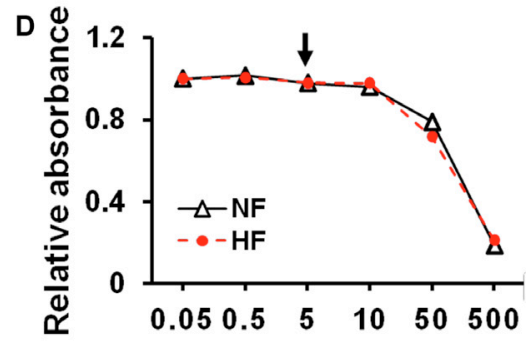

PSC833 concentration ( $\mu \mathrm{M})$

Figure 4: Evaluation of the resistance to verapamil after pre-treating cells with P-glycoprotein inhibitor in NF and $\mathrm{HF}$. (A) Cell density in NF and HF after Ver alone or with PSC833 pre-treatment was visualized under light microscope. (B) The living cell number in each treatment in (A) was counted and compared. (C) The dead cell rate in each treatment group was analyzed by flow cytometry and compared. Error bars represent means $\pm \mathrm{SD}(n=4),{ }^{* *} p<0.01$. (D) The cytotoxicity of PSC833 in NF and HF was determined by MTT assay with the absorbance measured at $540 \mathrm{~nm}$. PSC 833 at $5 \mu \mathrm{M}$ was the concentration used in this study as indicated by black arrow. 
in HS. HF were treated with latrunculin-A, an actin depolymerization agent, or DMSO for $12 \mathrm{~h}$ and lysed for immunoblotting. Results showed that latrunculin-A did not affect the protein level of P-glycoprotein or MRP1, while it significantly decreased $\beta$-actin level by $70 \%$ (Figure 7A). Notably, Co-IP analysis showed that latrunculin-A disrupted

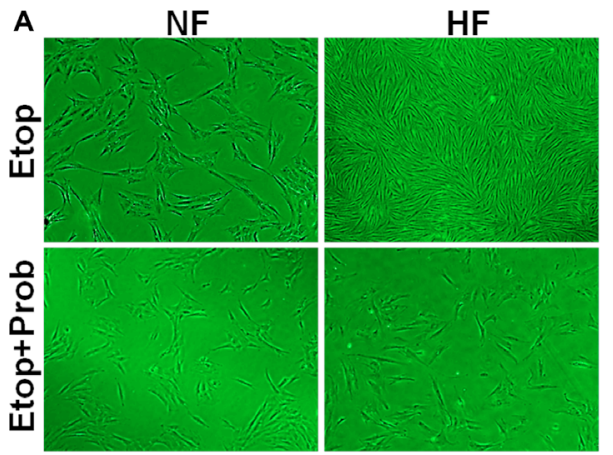

B

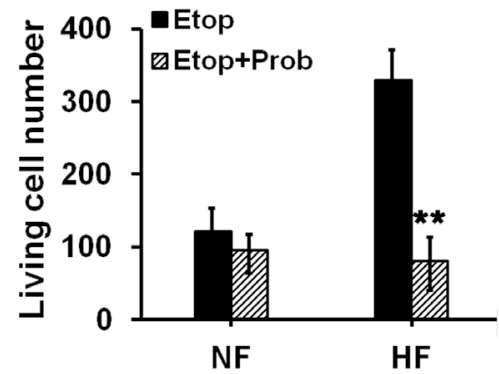

C
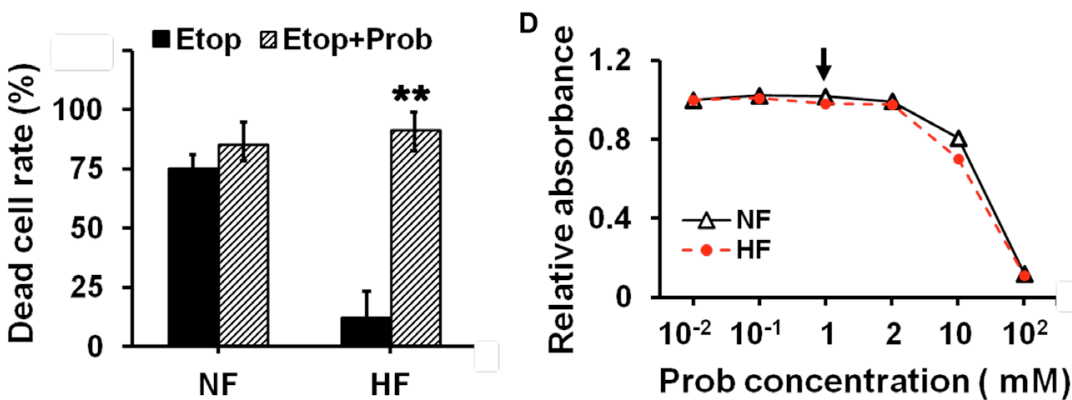

Figure 5: Evaluation of the resistance to etoposide phosphate after pre-treating cells with MRP1 inhibitor in NF and HF. (A) Cell density in NF and HF after Etop alone or with probenecid (Prob) pre-treatment was visualized under light microscope. (B) The living cell number under each treatment in (A) was counted and compared. (C) The dead cell rate in each treatment group was analyzed by flow cytometry and compared. Error bars represent means $\pm \mathrm{SD}(n=4), * * p<0.01$. (D) The cytotoxicity of Prob in NF and HF was determined by MTT assay with the absorbance measured at $540 \mathrm{~nm}$. Prob at $5 \mu \mathrm{M}$ was the concentration used in this study as indicated by black arrow.
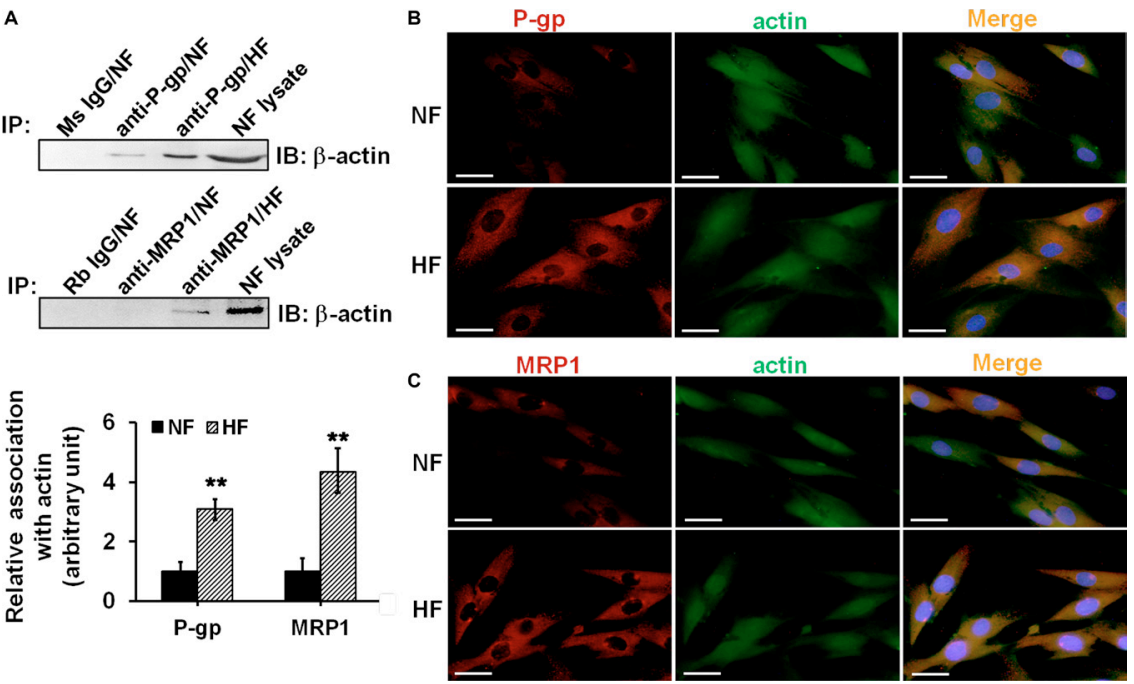

Figure 6: Study to assess the association and co-localization between P-glycoprotein/MRP1 with actin in NF and HF. (A) Co-IP was performed to assess the association between P-glycoprotein/MRP1 with actin in NF and HF. Mouse (Ms) or rabbit (Rb) IgG was used as negtive control for its corresponding primary antibody. Lysate from NF without Co-IP was used as positive control. The relative association of P-glycoprotein/MRP1 with actin in NF and HF was summarized and compared in histogram. Error bars represent means \pm $\mathrm{SD}(n=4),{ }^{* *} p<0.01$. (B-C) Dual-labeled immunofluorescent staining was used to assess the co-localization between P-glycoprotein (b, red) or MRP1(c, red) with actin (green). Nuclei were visualized with DAPI (blue). The co-localization was indicated in orange in merged images. Scale bar: $20 \mu \mathrm{m}$. 
the strong interaction of P-glycoprotein/actin by $\sim 90 \%$ and MRP1/actin by $\sim 70 \%$ in HF (Figure $7 \mathrm{~B}$ ). When HF were pre-treated with latrunculin-A for $12 \mathrm{~h}$ and then subjected to Ver or Etop for another $12 \mathrm{~h}$, the living cell number was reduced from $\sim 300$ to $\sim 50$ when compared to Ver or Etop treatment alone (Figure 7C-7D), suggesting latrunculinA-induced disruption of P-glycoprotein/MRP1-actin association abolished the drug resistance in HF.

\section{The use of specific anti-actin antibody abrogated drug resistance in hypertrophic scar fibroblasts}

An anti-actin antibody was used to specifically inhibit actin function. Briefly, HF were pre-treated with the antiactin or normal rabbit IgG (vehicle control) for $24 \mathrm{~h}$ and then subjected to Ver or Etop treatment for $12 \mathrm{~h}$, the living cell number was significantly reduced compared to Ver or Etop treatment alone (Figure 8A-8B), suggesting that the inhibition of actin function abolished drug resistance in $\mathrm{HF}$.

\section{DISCUSSION}

HS is featured by the overgrowth of dermal fibroblasts [12] and considered as a benign skin tumor. Thus, chemotherapy has been used for HS treatment.
Drug resistance is one of the major clinical challenges in cancer chemotherapy and often leads to the failure of chemotherapy and cancer recurrence [1-2]. Given the similarity between HS and tumor, it is reasonable to expect the existence of drug resistance phenomenon in HS or HS-derived fibroblasts. In this study, verapamil and etoposide phosphate, two agents with potential anti-cancer effect, were selected to investigate drug resistance in HS. As shown in Figure 1, normal skin fibroblasts were more sensitive to verapamil and etoposide, and exhibited less living cell number (Figure 1A-1B) and higher dead cell rate (Figure 1C-1D) than hypertrophic scar fibroblasts, suggesting the presence of drug resistance in HS.

Drug transporters play important roles in mediating the chemosensitivity and drug resistance in cancer cells [13]. P-glycoprotein and MRP1 are the two best characterized drug resistance-related transporters responsible for the failure of cancer chemotherapy [14]. They are often over-expressed in cancer cells and actively reduce intracellular drug accumulation below toxic concentration via pumping drugs out of cells [15-16]. Importantly, the resistance to verapamil or etoposide has been reported to be mediated by P-glycoprotein or MRP1, respectively [17]. Our study revealed significantly elevated expression of P-glycoprotein and MRP1 in scar fibroblasts,

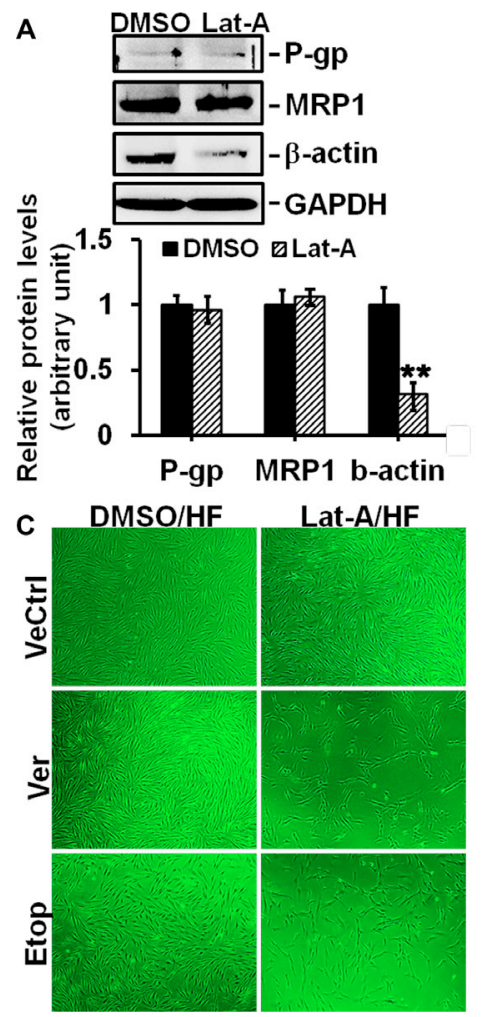

B
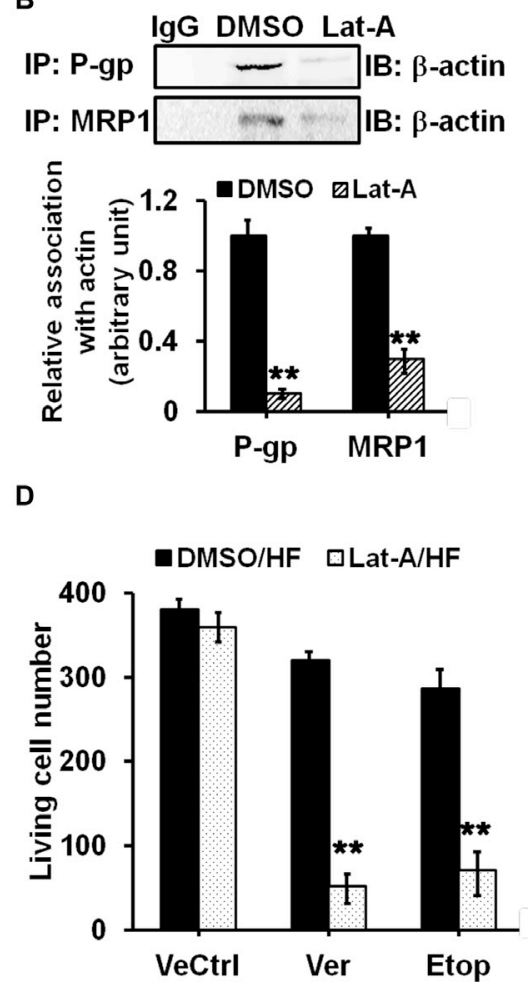

Figure 7: Effects of latrunculin-A on P-glycoprotein/MRP1-actin association and on drug resistance in $\mathrm{HF}$. (A) Immunoblotting to assess the effect of latrunculin-A (Lat-A) on protein level of P-glycoprotein, MRP1 or $\beta$-actin in HF. GAPDH served as an equal protein loading control. (B) Co-IP to assess the effect of Lat-A on P-glycoprotein/MRP1-actin association in HF. Normal mouse or rabbit IgG served as negtive control. (C) Cell density in HF after Ver or Etop treatment with/without Lat-A pre-treatment was visualized under light microscope. (D) The living cell number in each treatment group was counted and compared. Error bars represent means $\pm \mathrm{SD}$ $(n=6),{ }^{* *} p<0.01$. 
while only slight expression in normal fibroblasts (Figure 2B). Interestingly, although P-glycoprotein and MRP1 were reported to primarily locate at the plasma membrane in many epithelial and endothelial cells [18], our study showed that P-glycoprotein and MRP1 were predominantly distributed in the cytoplasm in cultured scar fibroblasts, with tiny staining in normal fibroblast cytoplasm (Figure 2A). In vivo studies further confirmed above observation, results showed that although normal skin dermis expressed little P-glycoprotein and MRP1, their protein levels were notably enhanced in hypertrophic scar dermis (Figure 3C). IHC on normal dermis and scar dermis revealed the tissue distribution of P-glycoprotein and MRP1. Due to the diversity on fibroblast density in normal dermis and scar dermis, the positivley-stained fibroblast rate was compared between two groups instead of the positively-stained cell number, results indicated significantly higher stained cell rate in scar dermis than that in normal dermis (Figure 3A-3B). Above data, on the other hand, demonstrate the existence of drug resistance in HS. Besides, PepT1, an oligopeptide transporter that does not confer drug resistance, showed no difference in its expression and distribution between normal skin and HS and served here as an internal control (Figures 2-3).

Inhibiting the function of drug transporter is a way to study drug resistance [19-20]. In order to elucidate whether the resistance to verapamil and etoposide in HS was confered by P-glycoprotein and MRP1, PSC 833 and probenecid were used to specifically inhibit the function of P-glycoprotein and MRP1 in cultured fibroblasts. Results showed that the resistance to verapamil or etoposide in scar fibroblasts was remarkably abolished when cells was pretreated with PSC833 or probenecid. (Figures 4-5). Also, pre-treating cells with PSC-833 or probenecid significantly decreased the living cell number (Figures $4 \mathrm{~A}-4 \mathrm{~B}$ and $5 \mathrm{~A}-5 \mathrm{~B}$ ) and increased the dead cell rate (Figures $4 \mathrm{C}-4 \mathrm{D}$ and $5 \mathrm{C}-5 \mathrm{D}$ ) in scar fibroblasts, while did not affect normal fibroblasts. These data demonstrate that drug resistance in HS is mediated by P-glycoprotein and MRP1.

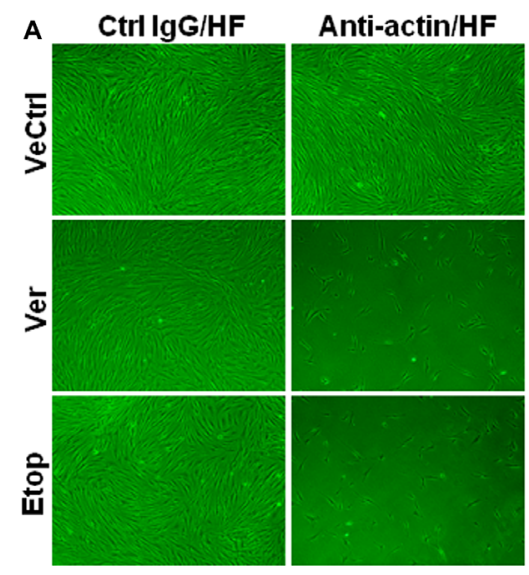

There are emerging evidences linking the function of drug transporters, such as P-glycoprotein, with actin microfilament system. A few reports have suggested the involvement of P-glycoprotein-actin association in drug resistance [21-22]. A recent study showed that cytochalasin, an actin-perturbing agent, enhanced intracellular drug accumulation in leukemia cells, indicating the attenuation of drug resistance in these cells [23]. Another similar study reported the disruption of actin stress-fiber network increased the intracellular drug accumulation and cytotoxic effect in osteosarcoma cells, suggesting the abrogation of drug resistance [9]. Our present study confirmed the structural interaction between P-glycoprotein and actin filament in skin fibroblasts by co-immunoprecipitation and immuno-colocalization, and for the first time revealed the association between MRP1 and actin (Figure 6). More importantly, the association and co-localization between P-glycoprotein/MRP-1 and actin were significantly elevated in scar fibroblasts than those in normal fibroblasts (Figure 6), suggesting a potential linkage between actin cytoskeletal network and the resistance in scar fibroblasts. When scar fibroblasts were treated with latrunculin-A, an actin cytoskeleton disrupting compound, only the steady-state level of $\beta$-actin was affected, but not P-glycoprotein and MRP1, suggesting the specificity of this inhibitor (Figure 7A). Also, latrunculin-A significantly abolished the association between P-glycoprotein/MRP1 and actin in scar fibroblasts (Figure 7B). When cells were pre-treated with latrunculin-A, the resistance to verapamil or etoposide in scar fibroblasts was remarkably attenuated (Figure 7C-7D). Moreover, the use of specific anti-actin antibody abrogated drug resistance in hypertrophic scar fibroblasts (Figure 8A-8B). These data make it conceivable that the linkage of drug transporter with actin cytoskeleton functions as a potential mechanism in the development of drug resistance in HS.

Taken together, this study reveals the presence of drug resistance in HS that is closely related to the enhanced

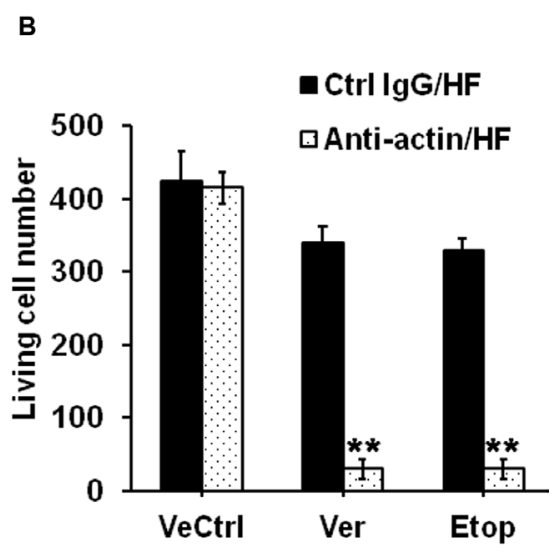

Figure 8: Effect of the specific anti-actin antibody on drug resistance in HF. (A) Cell density in HF after Ver or Etop treatment with/without the anti-actin antibody pre-treatment was visualized under light microscope. (B) The living cell number in each treatment group was counted and compared. Error bars represent means $\pm \mathrm{SD}(n=6), * * p<0.01$. 
expression of drug resistance-related transporters, such as P-glycoprotein and MRP1, defines for the first time an enhanced association between P-glycoprotein/ MRP1 and actin cytoskeleton in HS, and also discloses a dramatic reduction of P-glycoprotein/MRP1-mediated drug resistance when actin function was specifically inhibited. Research on new molecules or gene therapy strategies aiming at selectively disrupting drug transportercytoskeleton interaction or P-glycoprotein/MRP1mediated drug resistance, may stem from the results of this study to improve the efficacy of HS chemotherapy.

\section{MATERIALS AND METHODS}

\section{Ethics statement}

The protocol for studies using human samples was reviewed and approved by the Institutional Ethics Committee of the Fourth Military Medical University. Patients who offered skin samples including normal skin and hypertrophic scar provided their written, informed consent. Patient samples were deidentified and anonymized prior to analysis.

\section{Primary skin fibroblast isolation and culture}

Normal skin and hypertrophic scar tissues were obtained from sixteen patients (Table 1) who had been undergoing plastic surgery in Department of Burns and Cutaneous Surgery, Xijing Hospital (Xi'an, China). All specimens were collected with the permission from the Human Subjects Committee of the local institution. Dermal portions were minced and incubated in a solution of collagenase type I (Sigma-Aldrich, St. Louis, MO) at $0.1 \mathrm{mg} / \mathrm{ml}$ at $37^{\circ} \mathrm{C}$ for $3 \mathrm{~h}$ to separate fibroblasts. Isolated fibroblasts were then pelleted and grown in DMEM (Gibco, Grand Island, NY) supplemented with 10\% fetal calf serum (Gibco), $100 \mathrm{U} / \mathrm{ml}$ penicillin, and $100 \mathrm{U} / \mathrm{ml}$ streptomycin at $37^{\circ} \mathrm{C}$ in a humidified atmosphere with $5 \%(\mathrm{v} / \mathrm{v}) \mathrm{CO}_{2}$. Cells were seeded into six-well plates at $2 \times 10^{5}$ cells/well in normal growth medium and cells at the third and fourth sub-passages were used for experiments. Prior to drug treatment, cells were cultured in serum-free DMEM for $12 \mathrm{~h}$ and then treated with various drugs (detailed chemical information in Table 2). Cells in control group were added with an equal volume of serumfree medium with corresponding vehicle control. Cells were harvested and analyzed at time points indicated in each experiment.

\section{Immunoblotting}

Briefly, $50 \mu \mathrm{g}$ lysates from normal skin dermis, hypertrophic scar dermis, and their corresponding fibroblasts were subject to SDS-PAGE and transferred onto PVDF membranes (Millipore, Bedford, MA). After blocking with 5\% non-fat milk, membranes were incubated with specific primary antibodies (Table 3) at $4^{\circ} \mathrm{C}$ overnight. On the following day, membranes were washed and incubated with corresponding HRPconjugated secondary antibodies at $37^{\circ} \mathrm{C}$ for $1 \mathrm{~h}$. Proteins were visualized by enhanced chemiluminescence system using Fluor Chem FC (Alpha Innotech).

\section{Co-immunoprecipitation (Co-IP)}

Lysates from normal skin fibroblasts and hypertrophic scar fibroblasts were prepared in IP lysis buffer (10 mM Tris, $0.15 \mathrm{M} \mathrm{NaCl}, 1 \% \mathrm{NP}-40$, and $10 \%$ glycerol, $\mathrm{pH} 7.4$ at $22^{\circ} \mathrm{C}$ ) supplemented with protease and phosphatase inhibitor cocktails according to manufacturer's instruction. Co-IP was performed to identify if actin was structurally associated with P-glycoprotein or MRP1. In brief, $2 \mu \mathrm{g}$ mouse or rabbit IgG was added to $400 \mu \mathrm{g}$ lysates of fibroblasts and incubated for $1 \mathrm{~h}$ before precipitated with $10 \mu \mathrm{l}$ protein $\mathrm{A} / \mathrm{G}$ agarose beads (Santa Cruz Biotechnology, Santa Cruz, CA) for $1 \mathrm{~h}$, supernatant was then obtained after centrifuging at $1000 \mathrm{~g}$ for $5 \mathrm{~min}$. This pre-cleaning step removed non-specific interacting proteins from cell lysates. Thereafter, lysates were incubated with $2 \mu \mathrm{g}$ normal mouse/rabbit IgG as negative control or specific anti-P-glycoprotein or anti-MRP1 antibody for Co-IP on a Labnet MiniLabRoller overnight, to be followed by incubation with $20 \mu \mathrm{l}$ protein $\mathrm{A} / \mathrm{G}$ agarose beads to extract the immunocomplexes. Thereafter, beads were washed with IP lysis buffer and immunocomplexes (P-glycoprotein/MRP1 and its interacting protein partners) were extracted in SDS sample buffer at $100^{\circ} \mathrm{C}$ for SDSPAGE and immunoblot analysis. $50 \mu \mathrm{g}$ normal fibroblast lysates without IP served as the positive control.

\section{Immunocytofluorescence}

Fibroblasts were fixed in 4\% paraformaldehyde for 10 min. After washing with PBS, cells were permeabilized with $0.1 \%$ Triton-X 100 for $10 \mathrm{~min}$ and blocked with $1 \%$ bovine serum albumin at room temperature for $1 \mathrm{~h}$. Samples were then incubated with 1:50 diluted primary antibody at $4^{\circ} \mathrm{C}$ overnight at appropriate dilutions (Table 3). After being washed with PBS, samples were incubated with 1:100 diluted FITC-488 or Cy3-555 conjugated secondary antibody (Invitrogen, Carlsbad, CA) at $37^{\circ} \mathrm{C}$ for $1 \mathrm{~h}$. After being washed with PBS three times, samples were stained with DAPI for $30 \mathrm{~min}$. Coverslips were then mounted onto glass slides and viewed with Olympus Box-Type Photofluorography Unit Model FSX100 (Olympus, Japan). 
Table 1: The profile of each sample for primary culture

\begin{tabular}{lccccc}
\hline Sample & Gender & Age (years) & Biopsy site & Duration of lesion (months) & Etiology \\
\hline NF/HF 1 & Male & 17 & Cheek & 10 & Post-operation \\
NF/HF 2 & Male & 20 & Arm & 16 & Electric injury \\
NF/HF 3 & Male & 20 & Shoulder & 6 & Burn \\
NF/HF 4 & Male & 21 & Shoulder & 5 & Scald \\
NF/HF 5 & Male & 24 & Shoulder & 24 & Scald \\
NF/HF 6 & Male & 36 & Buttock & 7 & Burn \\
NF/HF 7 & Male & 38 & Chest & 17 & Post-operation \\
NF/HF 8 & Male & 42 & Chest & 15 & Post-operation \\
NF/HF 9 & Female & 16 & Chest & 8 & Trauma \\
NF/HF 10 & Female & 18 & Chest & 14 & Scald \\
NF/HF 11 & Female & 24 & Shoulder & 9 & Burn \\
NF/HF 12 & Female & 25 & Arm & 10 & Scald \\
NF/HF 13 & Female & 27 & Cheek & 12 & Burn \\
NF/HF 14 & Female & 43 & Shoulder & 11 & Burn \\
NF/HF 15 & Female & 45 & Back & 10 & Trauma \\
NF/HF 16 & Female & 46 & Back & 18 & Scald \\
\hline
\end{tabular}

Abbreviations: NF, normal skin fibroblast; HF, hypertrophic scar-derived fibroblast.

Table 2: Detailed information on the chemicals used in this study

\begin{tabular}{|lllllcc|}
\hline \multicolumn{1}{c}{ Chemicalnames } & \multicolumn{1}{c}{ Function } & \multicolumn{1}{c}{ Vendor } & Cat no. & Lot no. & $\begin{array}{c}\text { Working } \\
\text { concentration }\end{array}$ & Solution \\
\hline verapamil & P-glycoprotein substrate & Santa cruz biotechnology & sc-3590 & $152-11-4$ & $200 \mu \mathrm{M}$ & ethanol \\
\hline PSC833 & P-glycoprotein inhibitor & Santa cruz biotechnology & sc-361298 & $121584-18-7$ & $5 \mu \mathrm{M}$ & $\mathrm{DMSO}$ \\
\hline etoposide phosphate & MRP1 substrate & Santa cruz biotechnology & sc-357357 & $117091-64-2$ & $50 \mu \mathrm{M}$ & cold water \\
\hline probenecid & MRP1 inhibitor & Santa cruz biotechnology & sc-202773 & $57-66-9$ & $1 \mathrm{mM}$ & $\mathrm{DMSO}$ \\
\hline latrunculin-A & $\begin{array}{l}\text { actin depolymerization } \\
\text { reagent }\end{array}$ & Santa cruz biotechnology & sc-202691 & $76343-93-6$ & $0.2 \mu \mathrm{M}$ & $\mathrm{DMSO}$ \\
\hline
\end{tabular}

Table 3: Summary of primary antibodies used in this study

\begin{tabular}{|c|c|c|c|c|c|c|c|c|}
\hline \multirow[t]{2}{*}{ Antigen } & \multirow[t]{2}{*}{ Cat no. } & \multirow[t]{2}{*}{ Host } & \multirow[t]{2}{*}{ Vendor } & \multicolumn{5}{|c|}{ Dilution } \\
\hline & & & & IB & IHC & IF & IP & Inhibitor \\
\hline P-glycoprotein & sc-55510 & Mouse & Santa cruz biotechnology & $1: 200$ & $1: 50$ & $1: 50$ & $1: 40$ & \\
\hline MRP1 & ab24102 & Mouse & Abcam & $1: 200$ & & $1: 50$ & & \\
\hline MRP1 & sc-13960 & Rabbit & Santa cruz biotechnology & & $1: 50$ & & $1: 40$ & \\
\hline PepT1 & sc-20653 & Rabbit & Santa cruz biotechnology & $1: 200$ & $1: 50$ & & & \\
\hline Actin & sc-1616 & Goat & Santa cruz biotechnology & $1: 500$ & & & & \\
\hline Actin & 4967 & Rabbit & Cell signaling technology & & & $1: 100$ & & $1: 500$ \\
\hline
\end{tabular}

Abbreviations: IB, immunoblotting; IHC, immunohistochemistry; IF, immunofluorescence; IP, immunoprecipitation.

\section{Immunohistochemistry}

Hypertrophic scar and normal skin tissues were fixed in $10 \%$ buffered formalin solution overnight.
The 4- $\mu \mathrm{m}$ thickness paraffin-embedded sections were dewaxed, the endogenous peroxidase activity was quenched with $3 \%$ hydrogen peroxide for $15 \mathrm{~min}$, and then blocked for non-specific bindings by normal goat serum 
for $30 \mathrm{~min}$. Sections were then incubated overnight at $4^{\circ} \mathrm{C}$ with appropriate primary antibody dilutions (Table 3 ) and then followed by the application of PV-6000 Histostain TM Kit (ZSJQ, China). Briefly, sections were incubated with biotinylated secondary antibody, streptavidin-biotinhorseradish peroxidase was used for signal amplification and diaminobenzidine (DAB) for staining, sections were then counterstained with hematoxylin. Negative control was achieved by an isotypematched $\operatorname{IgG}$ in each of the immunostaining. Images were captured using an Olympus fluorescence microscope with an Olympus FSX100 digital camera. All images were subsequently compiled and analyzed using IPP (Image-Pro Plus) software package.

\section{Flow cytometry}

Cell death was evaluated by flow cytometry. Cells were treated with various drugs for specific period and washed with PBS. After washing, cells were resuspended in $100 \mu$ Annexin-V (diluted at 1:100 in 1\% BSA in PBS) added with $5 \mu \mathrm{l}$ FITC plus $5 \mu \mathrm{PI}$, and incubated for $15 \mathrm{~min}$ at room temperature in dark. $400 \mathrm{ul}$ Annexin-V was added to above mixture, cell death was then measured by BD FACSAria III flow cytometry (BD Bioscience, San Jose, CA).

\section{Cytotoxicity assay}

The cytotoxicity of PSC833 and probenecid was evaluated by MTT assay. In brief, fibroblasts were seeded in 96-well plates at $3 \times 10^{4}$ cells/well and kept overnight allowing full attachment. On the next day culture medium was replaced with fresh one supplemented with various concentrations of probenecid at $10^{-2}, 10^{-1}, 1,2,10$ and $10^{2} \mathrm{mM}$ or PSC833 at $0.05,0.5,5,10,50$ and $500 \mu \mathrm{M}$, cells were allowed to grow for $48 \mathrm{~h}$. Then $100 \mu \mathrm{l}$ MTT $(10 \mathrm{mg} / \mathrm{ml})$ was added to each well $4 \mathrm{~h}$ before completion of this incubation. Thereafter, $100 \mu \mathrm{l}$ DMSO was added to each well. The optical density (OD) was measured at $540 \mathrm{~nm}$ with a Bio-Rad Model 680 Plate Reader (Bio-Rad Laboratories, Hercules, CA).

\section{Statistical analysis}

Each experiment reported herein was repeated at least three to four times, excluding pilot experiments. Statistical analysis was performed using the GB-STAT software package (version 7.0; Dynamic Microsystems, Silver Spring, MD). One-way ANOVA was performed and followed by Dunnett's test for multiple comparisons. In selected experiments, Student's $t$ test was used for paired comparisons.

\section{ACKNOWLEDGMENTS}

None.

\section{CONFLICTS OF INTEREST}

The authors declare no competing financial interests.

\section{FUNDING}

This work was supported by Xijing Hospital Natural Science Funds for Distinguished Young Scientists (No: XJZT14J06 to L.S.).

\section{Authors' contributions}

L.S. designed the research and wrote the manuscript; L.S., L.F. and Y.L. performed experiments, analyzed data and revised the manuscript; F.Y. and M.Z. contributed reagents, materials and analysis tools; D.H. and L.S. approved the final submission. All authors discussed the results and reviewed the manuscript.

\section{REFERENCES}

1. Gottesman MM, Fojo T, Bates SE. Multidrug resistance in cancer: role of ATP-dependent transporters. Nat Rev Cancer. 2002; 2:48-58.

2. Donnenberg VS, Donnenberg AD. Multiple drug resistance in cancer revisited: the cancer stem cell hypothesis. J Clin Pharmacol. 2005; 45:872-877.

3. Choi YH, Yu AM. ABC Transporters in Multidrug Resistance and Pharmacokinetics, and Strategies for Drug Development. Curr Pharm Des. 2014; 20:793-807.

4. Chang XB. A molecular understanding of ATP-dependent solute transport by multidrug resistance-associated protein MRP1. Cancer Metastasis Rev. 2007; 26:15-37.

5. Luna EJ, Hitt AL. Cytoskeleton-plasma membrane interactions. Science. 1992; 258:955-964.

6. Kusumi A, Sako Y. Cell surface organization by the membrane skeleton. Curr Opin Cell Biol. 1996; 8:566-574.

7. Wang Z, Chen J, Wang J, Ahn S, Li CM, Lu Y, Loveless VS, Dalton JT, Miller DD, Li W. Novel tubulin polymerization inhibitors overcome multidrug resistance and reduce melanoma lung metastasis. Pharm Res. 2012; 29:3040-3052.

8. Jorfi S, Inal JM. The role of microvesicles in cancer progression and drug resistance. Biochem Soc Trans. 2013; 41:293-298.

9. Takeshita H, Kusuzaki K, Ashihara T, Gebhardt MC, Mankin HJ, Hirasawa Y. Actin organization associated with the expression of multidrug resistant phenotype in osteosarcoma cells and the effect of actin depolymerization on drug resistance. Cancer Lett. 1998; 126:75-81.

10. Bichat F, Mouawad R, Solis-Recendez G, Khayat D, Bastian G. Cytoskeleton alteration in MCF7R cells, a multidrug resistant human breast cancer cell line. Anticancer Res. 1997; 17:3393-3401. 
11. Luciani F, Molinari A, Lozupone F, Calcabrini, Lugini L, Stringer A, Puddu P, Arancia G, Cianfriglia M, Fais S. P-glycoprotein-actin association through ERM family proteins: a role in P-glycoprotein function in human cells of lymphoid origin. Blood. 2002; 99:641-648.

12. Singer AJ, Clark RA. Cutaneous wound healing. N Engl J Med. 1999; 341:738-746.

13. Huang Y, Sadée W. Membrane transporters and channels in chemoresistance and -sensitivity of tumor cells. Cancer Lett. 2006; 239:168-182.

14. Childs S, Ling V. The MDR superfamily of genes and its biological implications. Important Adv Oncol. 1994: 21-36.

15. Huang Y. Pharmacogenetics/genomics of membrane transporters in cancer chemotherapy. Cancer Metastasis Rev. 2007; 26:183-201.

16. Vander Borght S, Komuta M, Libbrecht L, Katoonizadeh A, Aerts R, Dymarkowski S, Verslype C, Nevens F, Roskams T. Expression of multidrug resistance-associated protein 1 in hepatocellular carcinoma is associated with a more aggressive tumour phenotype and may reflect a progenitor cell origin. Liver International. 2008; 28: 1370-1380.

17. Römermann K, Wanek T, Bankstahl M, Bankstahl JP, Fedrowitz M, Müller M, Löscher W, Kuntner C, Langer O. (R)-[(11)C]verapamil is selectively transported by murine and human P-glycoprotein at the blood-brain barrier, and not by MRP1 and BCRP. Nucl Med Biol. 2013; 40:873-878.

18. Porcelli L, Lemos C, Peters GJ, Paradiso A, Azzariti A. Intracellular trafficking of MDR transporters and relevance of SNPs. Curr Top Med Chem. 2009; 9:197-208.

19. Binkhathlan Z, Lavasanifar A. P-glycoprotein inhibition as a therapeutic approach for overcoming multidrug resistance in cancer: current status and future perspectives. Curr Cancer Drug Targets. 2013; 13:326-346.

20. Callaghan R, Luk F, Bebawy M. Inhibition of the multidrug resistance P-glycoprotein: time for a change of strategy? Drug Metab Dispos. 2014; 42:623-631.

21. Capella LS, Alcantara JS, Moura-Neto V, Lopes AG, Capella MA. Vanadate is toxic to adherent- growing multidrug-resistant cells. Tumour Biol. 2000; 21:54-62.

22. Erokhina MV, Shtil AA, Shushanov SS, Sidorova TA, Stavrovskaya AA. Partial restoration of the actin cytoskeleton in transformed Syrian hamster fibroblasts selected for low levels of 'typical' multidrug resistance. FEBS Lett. 1994; 341:295-298.

23. Tsuruo T, Iida H. Effects of cytochalasins and colchicine on the accumulation and retention of daunomycin and vincristine in drug resistant tumor cells. Biochem Pharmacol. 1986; 35:1087-1090. 